ChangXing Miao (Beijing)

Youbin Zhu (Xi'an)

\title{
INVARIANTS, CONSERVATION LAWS AND TIME DECAY FOR A NONLINEAR SYSTEM OF KLEIN-GORDON EQUATIONS WITH HAMILTONIAN STRUCTURE
}

Abstract. We discuss invariants and conservation laws for a nonlinear system of Klein-Gordon equations with Hamiltonian structure

$$
\left\{\begin{array}{l}
u_{t t}-\Delta u+m^{2} u=-F_{1}\left(|u|^{2},|v|^{2}\right) u \\
v_{t t}-\Delta v+m^{2} v=-F_{2}\left(|u|^{2},|v|^{2}\right) v
\end{array}\right.
$$

for which there exists a function $F(\lambda, \mu)$ such that

$$
\frac{\partial F(\lambda, \mu)}{\partial \lambda}=F_{1}(\lambda, \mu), \quad \frac{\partial F(\lambda, \mu)}{\partial \mu}=F_{2}(\lambda, \mu) .
$$

Based on Morawetz-type identity, we prove that solutions to the above system decay to zero in local $L^{2}$-norm, and local energy also decays to zero if the initial energy satisfies

$$
\begin{aligned}
E\left(u, v, \mathbb{R}^{n}, 0\right)= & \frac{1}{2} \int_{\mathbb{R}^{n}}\left(|\nabla u(0)|^{2}+\left|u_{t}(0)\right|^{2}+m^{2}|u(0)|^{2}+|\nabla v(0)|^{2}\right. \\
& \left.+\left|v_{t}(0)\right|^{2}+m^{2}|v(0)|^{2}+F\left(|u(0)|^{2},|v(0)|^{2}\right)\right) d x<\infty,
\end{aligned}
$$

and

$$
\begin{aligned}
& F_{1}\left(|u|^{2},|v|^{2}\right)|u|^{2}+F_{2}\left(|u|^{2},|v|^{2}\right)|v|^{2}-F\left(|u|^{2},|v|^{2}\right) \\
& \quad \geq a F\left(|u|^{2},|v|^{2}\right) \geq 0, \quad a>0 .
\end{aligned}
$$

1. Introduction. In her seminal paper [5] Morawetz established a radial identity for Klein-Gordon equations; for Schrödinger equations a similar identity was obtained by Lin and Strauss in [4]. Morawetz's radial iden-

2000 Mathematics Subject Classification: 35L05, 35L15.

Key words and phrases: Klein-Gordon equations, variational principle, local energy, Lagrange density function. 
tity, like other invariants and conservation laws, plays an important role in the scattering theory of nonlinear Klein-Gordon equations and nonlinear Schrödinger equations (see $[1,2,6,7,9]$ for Klein-Gordon equations and [3, $4,7,9]$ for Schrödinger equations). W. Strauss derived the so-called multiplier identity for solutions of elliptic equations and Klein-Gordon equations based on the multiplier method in his pioneering paper [8] (see also [9]). The multiplier identity essentially provides many useful identities which include many conservation laws and Morawetz's radial identity.

In the Remark of Morawetz's famous paper [5], she mentioned the system

$$
\left\{\begin{array}{l}
\phi_{t t}-\Delta \phi+m^{2} \phi+g^{2} \psi^{2} \phi=0 \\
\psi_{t t}-\Delta \psi+m^{2} \psi+h^{2} \phi^{2} \psi=0
\end{array}\right.
$$

which represents the interaction of two scalar fields. In the present paper, we consider the following Klein-Gordon system with Hamiltonian structure:

$$
\left\{\begin{array}{l}
u_{t t}-\Delta u+m^{2} u=-F_{1}\left(|u|^{2},|v|^{2}\right) u \\
v_{t t}-\Delta v+m^{2} v=-F_{2}\left(|u|^{2},|v|^{2}\right) v
\end{array}\right.
$$

where there exists a function $F(\lambda, \mu)$ such that

$$
\frac{\partial F(\lambda, \mu)}{\partial \lambda}=F_{1}(\lambda, \mu), \quad \frac{\partial F(\lambda, \mu)}{\partial \mu}=F_{2}(\lambda, \mu) .
$$

Though it is not obvious, (1.2) is more general than (1.1). In fact, taking $u=c_{1} \phi, v=c_{2} \psi$ with $c_{2}^{2} g^{2}=c_{1}^{2} h^{2}=c,(1.1)$ becomes

$$
\left\{\begin{array}{l}
u_{t t}-\Delta u+m^{2} u=-c v^{2} u \\
v_{t t}-\Delta v+m^{2} v=-c u^{2} v
\end{array}\right.
$$

which is a special case of (1.2) with $F_{1}=c v^{2}$ and $F_{2}=c u^{2}$.

In this paper we have two purposes. One is to derive a multiplier identity for nonlinear systems of elliptic equations or Klein-Gordon equations with Hamiltonian structure by applying a variational principle. The other is to prove that smooth solutions of the Klein-Gordon system decay to zero in local $L^{2}$-norm, and their local energy also decays to zero, which is based on Morawetz's estimate obtained in our paper.

2. A Morawetz-Pohozaev identity for nonlinear systems of Klein-Gordon equations with Hamiltonian structure. In this section we consider nonlinear systems of elliptic equations with Hamiltonian structure

$$
\begin{cases}\Delta u=G_{1}\left(|u|^{2},|v|^{2}\right) u, & x \in \mathbb{R}^{N}, \\ \Delta v=G_{2}\left(|u|^{2},|v|^{2}\right) v, & x \in \mathbb{R}^{N}\end{cases}
$$


where there exists a function $G(\lambda, \mu)$ such that

$$
\frac{\partial G}{\partial \lambda}(\lambda, \mu)=G_{1}(\lambda, \mu), \quad \frac{\partial G}{\partial \mu}(\lambda, \mu)=G_{2}(\lambda, \mu) .
$$

Motivated by the pioneer paper of Strauss [8], we first derive the so-called multiplier identity for smooth solutions of (2.1), which provides many useful identities including conservation laws under the action of conformal groups and other transformation groups. In particular, we can derive the well known Pohozaev identity for (2.1) by taking the special multiplier

$$
M u=\frac{\partial u}{\partial r}+\frac{N-1}{2 r} u,
$$

i.e. the skew-adjoint part of the radial derivative $u_{r}$.

It is well known that in the relativistic case (2.1) can be reduced to the nonlinear Klein-Gordon system with Hamiltonian structure, so we can derive conservation laws and some useful identities by coordinate transformations. As a special example, we obtain the Morawetz-Pohozaev identity.

Consider the Lagrange density function associated with (2.1) and (2.2),

$$
\ell(u, v)=\frac{1}{2}\left[|\nabla u|^{2}+|\nabla v|^{2}+G\left(|u|^{2},|v|^{2}\right)\right] .
$$

Using the variational principle we can derive the following multiplier identity.

Proposition 2.1. Assume that $(u(x), v(x))$ is a smooth solution of (2.1), $M u=h(x) \cdot \nabla u+q(x) u, M v=h(x) \cdot \nabla v+q(x) v$, where $h(x)=\left(h_{1}(x), \ldots\right.$ $\left.\ldots, h_{N}(x)\right)$ is a vector function and $q(x)$ a scalar function. Set

$$
\begin{aligned}
& \operatorname{Eq}_{1}(u, v)=-\Delta u+G_{1}\left(|u|^{2},|v|^{2}\right) u, \\
& \operatorname{Eq}_{2}(u, v)=-\Delta v+G_{2}\left(|u|^{2},|v|^{2}\right) v .
\end{aligned}
$$

Then we have the following multiplier identity:

$$
\begin{aligned}
\mathrm{Eq}_{1}(u, v) M u+\mathrm{Eq}_{2}(u, v) M v & \\
= & -\nabla \cdot\langle\nabla u, h \cdot \nabla u+q u\rangle-\nabla \cdot\langle\nabla v, h \cdot \nabla v+q v\rangle+\nabla \cdot(h \ell(u, v)) \\
& +\frac{1}{2} \nabla \cdot\left(|u|^{2} \nabla q+|v|^{2} \nabla q\right)-\frac{1}{2} \Delta q\left(|u|^{2}+|v|^{2}\right)+\langle\nabla u, \nabla h \nabla u\rangle \\
& +\langle\nabla v, \nabla h \nabla v\rangle+(2 q-\nabla \cdot h) \ell(u, v)+q \widetilde{G}\left(|u|^{2},|v|^{2}\right),
\end{aligned}
$$

where $\langle a, b\rangle$ denotes ab in $\mathbb{R}, \operatorname{Re}(a \bar{b})$ in $\mathbb{C},\langle\nabla f, \nabla h \nabla f\rangle=\sum_{i, j} \partial_{i} f \partial_{i} h_{j} \partial_{j} f$ and

$$
\widetilde{G}\left(|u|^{2},|v|^{2}\right)=G_{1}\left(|u|^{2},|v|^{2}\right)|u|^{2}+G_{2}\left(|u|^{2},|v|^{2}\right)|v|^{2}-G\left(|u|^{2},|v|^{2}\right) .
$$

Proof. (2.4) can be shown by direct computation, but we would like to prove it by a variational method for clarity. Without loss of generality, we prove (2.4) for real-valued functions. 
SteP 1. Considering the variation of the Lagrange density function $\ell(u, v)$, we have

$$
\begin{aligned}
\delta_{w_{1}, w_{2}} \ell & (u, v)=\lim _{\varepsilon \rightarrow 0} \frac{\ell\left(u+\varepsilon w_{1}, v+\varepsilon w_{2}\right)-\ell(u, v)}{\varepsilon} \\
= & \left\langle-\Delta u+G_{1}\left(|u|^{2},|v|^{2}\right) u, w_{1}\right\rangle+\left\langle-\Delta v+G_{2}\left(|u|^{2},|v|^{2}\right) v, w_{2}\right\rangle \\
& +\nabla \cdot\left\langle\nabla u, w_{1}\right\rangle+\nabla \cdot\left\langle\nabla v, w_{2}\right\rangle \\
= & \mathrm{Eq}_{1}(u, v) w_{1}+\mathrm{Eq}_{2}(u, v) w_{2} \\
& +\nabla \cdot\left\langle\nabla u, w_{1}\right\rangle+\nabla \cdot\left\langle\nabla v, w_{2}\right\rangle .
\end{aligned}
$$

Let $T(\lambda)$ be a continuous transformation group in $\mathbb{R}^{N}$, and denote by $A$ its generator. By the variational principle it follows that

$$
\begin{aligned}
\lim _{\lambda \rightarrow 0} \frac{\ell(T(\lambda) u, T(\lambda) v)-\ell(u, v)}{\lambda} & =\lim _{\lambda \rightarrow 0} \frac{\ell(u+\lambda A u, v+\lambda A v)-\ell(u, v)}{\lambda} \\
& =\delta_{A u, A v} \ell(u, v) .
\end{aligned}
$$

This together with (2.5) implies that

$$
\begin{aligned}
\lim _{\lambda \rightarrow 0} \frac{\ell(T(\lambda) u, T(\lambda) v)-\ell(u, v)}{\lambda} & =\operatorname{Eq}_{1}(u, v) A u+\mathrm{Eq}_{2}(u, v) A v+\nabla \cdot\langle\nabla u, A u\rangle+\nabla \cdot\langle\nabla v, A v\rangle .
\end{aligned}
$$

STEP 2. Let $T(\lambda)$ be a continuous transformation group which satisfies

$$
\ell(T(\lambda) u, T(\lambda) v)=T(\lambda) \ell(u, v) .
$$

Taking the derivative with respect to $\lambda$ of both sides of the above identity, it follows from (2.6) that

$$
\mathrm{Eq}_{1}(u, v) A u+\mathrm{Eq}_{2}(u, v) A v=A \ell(u, v)-\nabla \cdot\langle\nabla u, A u\rangle-\nabla \cdot\langle\nabla v, A v\rangle .
$$

In particular, for the translation group in the space variable $x_{j}$, we have $A=\partial_{j}(1 \leq j \leq N)$, therefore

$$
\mathrm{Eq}_{1}(u, v) \partial_{j} u+\mathrm{Eq}_{2}(u, v) \partial_{j} v=\partial_{j} \ell(u, v)-\nabla \cdot\left\langle\nabla u, \partial_{j} u\right\rangle-\nabla \cdot\left\langle\nabla v, \partial_{j} v\right\rangle,
$$
i.e.,

$$
\mathrm{Eq}_{1}(u, v) \nabla u+\mathrm{Eq}_{2}(u, v) \nabla v=\nabla \ell(u, v)-\nabla \cdot\langle\nabla u, \nabla u\rangle-\nabla \cdot\langle\nabla v, \nabla v\rangle .
$$

On the other hand, set $T(\lambda) u=e^{\lambda} u$, so that $A=I$. For any function $\tilde{\ell}(u, v)$ with

$$
\widetilde{\ell}(T(\lambda) u, T(\lambda) v)=T(2 \lambda) \widetilde{\ell}(u, v),
$$

we obtain

$$
\delta_{u, v} \tilde{\ell}(u, v)=2 \widetilde{\ell}(u, v),
$$

by taking the derivative in $\lambda$ on both sides of (2.8). It is easy to verify that $\widetilde{\ell}=\ell-\frac{1}{2} G$ satisfies (2.8). For a general Lagrange density function $\ell(u, v)$, 
one has

$$
\begin{aligned}
\delta_{u, v} \ell(u, v) & =\delta_{u, v}\left(\tilde{\ell}(u, v)+\frac{1}{2} G\left(|u|^{2},|v|^{2}\right)\right) \\
& =2 \widetilde{\ell}(u, v)+G_{1}\left(|u|^{2},|v|^{2}\right)|u|^{2}+G_{2}\left(|u|^{2},|v|^{2}\right)|v|^{2} \\
& =2 \ell(u, v)+\widetilde{G}\left(|u|^{2},|v|^{2}\right)
\end{aligned}
$$

by (2.9). Thus we conclude from (2.6) that

$$
\begin{aligned}
\operatorname{Eq}_{1}(u, v) u+\operatorname{Eq}_{2}(u, v) v= & 2 \ell(u, v)+\widetilde{G}\left(|u|^{2},|v|^{2}\right) \\
& -\nabla \cdot\langle\nabla u, u\rangle-\nabla \cdot\langle\nabla v, v\rangle .
\end{aligned}
$$

STEP 3. From (2.7), we conclude that

$$
\begin{aligned}
& \left\langle\mathrm{Eq}_{1}(u, v), h \cdot \nabla u\right\rangle+\left\langle\mathrm{Eq}_{2}(u, v), h \cdot \nabla v\right\rangle \\
& =\nabla \cdot(h \ell(u, v))-(\nabla \cdot h) \ell(u, v)-\nabla \cdot\langle\nabla u, h \cdot \nabla u\rangle \\
& \quad-\nabla \cdot\langle\nabla v, h \cdot \nabla v\rangle+\langle\nabla u, \nabla h \nabla u\rangle+\langle\nabla v, \nabla h \nabla v\rangle
\end{aligned}
$$

by simple computation. On the other hand, in view of (2.10), it follows that $(2.12)$

$$
\begin{aligned}
\left\langle\mathrm{Eq}_{1}(u, v), q u\right\rangle+\left\langle\mathrm{Eq}_{2}(u, v), q v\right\rangle & \\
= & 2 q \ell(u, v)+q \widetilde{G}\left(|u|^{2},|v|^{2}\right)-\nabla \cdot\langle\nabla u, q u\rangle \\
& -\nabla \cdot\langle\nabla v, q v\rangle+\nabla q \cdot\langle\nabla u, u\rangle+\nabla q \cdot\langle\nabla v, v\rangle \\
= & 2 q \ell(u, v)+q \widetilde{G}\left(|u|^{2},|v|^{2}\right)-\nabla \cdot\langle\nabla u, q u\rangle-\nabla \cdot\langle\nabla v, q v\rangle \\
& +\frac{1}{2} \nabla \cdot\left(\left(|u|^{2}+|v|^{2}\right) \nabla q\right)-\frac{1}{2} \Delta q\left(|u|^{2}+|v|^{2}\right) .
\end{aligned}
$$

Combining (2.11) with (2.12), we show that (2.4) holds.

Choosing the multiplier $M u=h \cdot \nabla u+q u$ to be the skew-adjoint part of the radial derivative $u_{r}$, i.e.

$$
h(x)=\frac{x}{r}, \quad q(x)=\frac{N-1}{2 r}, \quad r=|x|,
$$

a simple computation implies that

$$
\begin{aligned}
& \partial_{i} h_{j}=\frac{\delta_{i j}}{r}-\frac{x_{i} x_{j}}{r^{3}}, \quad \nabla \cdot h=\sum_{i=1}^{N} \frac{\partial h_{i}}{\partial x_{i}}=\frac{N-1}{r}, \\
& \nabla q=\frac{N-1}{2} \nabla\left(\frac{1}{r}\right)=-\frac{N-1}{2} \frac{x}{r^{3}}, \\
& \Delta q=\frac{N-1}{2} \Delta\left(\frac{1}{r}\right)=-\frac{(N-1)(N-3)}{2 r^{3}}, \quad N \geq 4, \\
& \Delta q=\Delta\left(\frac{1}{r}\right)=-4 \pi \delta(x), \quad N=3 .
\end{aligned}
$$


As an immediate consequence of Proposition 2.1, we easily get the following Pohozaev identity for (2.1) from (2.13)-(2.17) by using the divergence theorem.

Corollary 2.2. Let $N \geq 3$. Assume that $(u(x), v(x))$ is a smooth solution of (2.1), and decays at $|x|=\infty$. Then for $N \geq 4$,

$$
\begin{aligned}
& \frac{(N-1)(N-3)}{4} \int_{\mathbb{R}^{N}} \frac{|u|^{2}+|v|^{2}}{r^{3}} d x \\
& \quad+\int_{\mathbb{R}^{N}} \frac{|\nabla u|^{2}-\left|u_{r}\right|^{2}}{r} d x+\int_{\mathbb{R}^{N}} \frac{|\nabla v|^{2}-\left|v_{r}\right|^{2}}{r} d x \\
& \quad+\frac{N-1}{2} \int_{\mathbb{R}^{N}} \frac{G_{1}\left(|u|^{2},|v|^{2}\right)|u|^{2}+G_{2}\left(|u|^{2},|v|^{2}\right)|v|^{2}-G\left(|u|^{2},|v|^{2}\right)}{r} d x=0,
\end{aligned}
$$

while for $N=3$,

$$
\begin{aligned}
2 \pi|u(0)|^{2}+ & 2 \pi|v(0)|^{2}+\int_{\mathbb{R}^{3}} \frac{|\nabla u|^{2}-\left|u_{r}\right|^{2}}{r} d x+\int_{\mathbb{R}^{3}} \frac{|\nabla v|^{2}-\left|v_{r}\right|^{2}}{r} d x \\
& +\int_{\mathbb{R}^{3}} \frac{G_{1}\left(|u|^{2},|v|^{2}\right)|u|^{2}+G_{2}\left(|u|^{2},|v|^{2}\right)|v|^{2}-G\left(|u|^{2},|v|^{2}\right)}{r} d x=0 .
\end{aligned}
$$

If we choose different conformal groups as the multiplier operator, we obtain

Corollary 2.3. Let $N \geq 3$. Assume that $(u(x), v(x))$ is a smooth solution of (2.1), and decays at $|x|=\infty$.

(i) (Translation) Setting $M=\partial_{j} \triangleq \partial / \partial x_{j}, j=1, \ldots, N$, yields

$$
\begin{aligned}
0= & \left\{-\left(\partial_{j} u\right)^{2}-\left(\partial_{j} v\right)^{2}+\frac{1}{2}\left[|\nabla u|^{2}+|\nabla v|^{2}+G\left(|u|^{2},|v|^{2}\right)\right]\right\}_{j} \\
& +\sum_{k \neq j}\left\{-\partial_{j} u \partial_{k} u-\partial_{j} v \partial_{k} v\right\}_{k},
\end{aligned}
$$

where we denote by $\{\cdot\}_{j}$ the derivative with respect to $x_{j}$.

(ii) (Rotation) Setting $M=x_{k} \partial_{j}-x_{j} \partial_{k}, 1 \leq j, k \leq N$ with $j \neq k$ gives

$$
\begin{aligned}
0= & \nabla \cdot\left\{-\left(x_{k} \partial_{j}-x_{j} \partial_{k}\right) u \nabla u\right\}+\nabla \cdot\left\{-\left(x_{k} \partial_{j}-x_{j} \partial_{k}\right) v \nabla v\right\} \\
& +\frac{1}{2}\left\{x_{k}\left(|\nabla u|^{2}+|\nabla v|^{2}+G\left(|u|^{2},|v|^{2}\right)\right)\right\}_{j} \\
& -\frac{1}{2}\left\{x_{j}\left(|\nabla u|^{2}+|\nabla v|^{2}+G\left(|u|^{2},|v|^{2}\right)\right)\right\}_{k} .
\end{aligned}
$$


(iii) (Dilation) If we set $M=x \cdot \nabla+(N-2) / 2$, we obtain

$$
\begin{aligned}
0= & \frac{N-2}{2}\left[G_{1}\left(|u|^{2},|v|^{2}\right)|u|^{2}+G_{2}\left(|u|^{2},|v|^{2}\right)|v|^{2}\right]-\frac{N}{2} G\left(|u|^{2},|v|^{2}\right) \\
& +\nabla \cdot\left\{-(x \cdot \nabla u) \nabla u-(x \cdot \nabla v) \nabla v+\frac{1}{2} x\left(|\nabla u|^{2}+|\nabla v|^{2}\right)\right. \\
& \left.-\frac{N-2}{2} u \nabla u-\frac{N-2}{2} v \nabla v+\frac{1}{2} x G\left(|u|^{2},|v|^{2}\right)\right\} .
\end{aligned}
$$

(iv) (Inversion) Setting

$$
M u=\left(x_{k}^{2}-\sum_{j \neq k} x_{j}^{2}\right) \partial_{k} u+2 x_{k} \sum_{j \neq k} x_{j} \partial_{j} u+(N-2) x_{k} u, \quad 1 \leq k \leq N,
$$

yields

$$
\begin{aligned}
0= & (N-2) x_{k}\left[G_{1}\left(|u|^{2},|v|^{2}\right)|u|^{2}+G_{2}\left(|u|^{2},|v|^{2}\right)|v|^{2}\right]-N x_{k} G\left(|u|^{2},|v|^{2}\right) \\
& +\nabla \cdot\left\{-\left[\left(x_{k}^{2}-\sum_{j \neq k} x_{j}^{2}\right) \partial_{k} u+2 x_{k} \sum_{j \neq k} x_{j} \partial_{j} u+(N-2) x_{k} u\right] \nabla u\right\} \\
& +\nabla \cdot\left\{-\left[\left(x_{k}^{2}-\sum_{j \neq k} x_{j}^{2}\right) \partial_{k} v+2 x_{k} \sum_{j \neq k} x_{j} \partial_{j} v+(N-2) x_{k} v\right] \nabla v\right\} \\
& +\frac{1}{2}\left\{\left(x_{k}^{2}-\sum_{j \neq k} x_{j}^{2}\right)\left(|\nabla u|^{2}+|\nabla v|^{2}+G\left(|u|^{2},|v|^{2}\right)\right)\right\}_{k} \\
& +\frac{1}{2} \sum_{j \neq k}\left\{2 x_{k} x_{j}\left(|\nabla u|^{2}+|\nabla v|^{2}+G\left(|u|^{2},|v|^{2}\right)\right)\right\}_{j} \\
& +\left\{\frac{N-2}{2}|u|^{2}+\frac{N-2}{2}|v|^{2}\right\}_{k},
\end{aligned}
$$

where the fact that $h_{k}=x_{k}^{2}-\sum_{j \neq k} x_{j}^{2}, h_{j}=2 x_{k} x_{j}, j \neq k$, has been used.

Now we derive the multiplier identity for a nonlinear Klein-Gordon system with Hamiltonian structure, i.e., (1.2) and (1.3).

Set $N=n+1$, and make the following coordinate and function changes:

$$
\begin{aligned}
& x \mapsto\left(x_{1}, \ldots, x_{n}, i t\right), \quad x_{N}=x_{n+1}=i t, \\
& G_{1}\left(|u|^{2},|v|^{2}\right)=m^{2}+F_{1}\left(|u|^{2},|v|^{2}\right), \quad G_{2}\left(|u|^{2},|v|^{2}\right)=m^{2}+F_{2}\left(|u|^{2},|v|^{2}\right) .
\end{aligned}
$$

Then (2.1) and (2.2) reduce to (1.2) and (1.3). Note that

$$
\partial_{n+1} \mapsto-i \partial_{t}, \quad \nabla \mapsto\left(\nabla,-i \partial_{t}\right)
$$

together with

$$
\begin{aligned}
h(x) & \mapsto \widetilde{h}=\left(h, i h_{n+1}\right)=\left(h_{1}(x, t), \ldots, h_{n}(x, t), i h_{n+1}\right), \\
M u & \mapsto M u=\widetilde{h} \cdot\left(\nabla,-i \partial_{t}\right) u+q u=h \cdot \nabla u+h_{n+1} \partial_{t} u+q u,
\end{aligned}
$$


and

$$
\begin{aligned}
\ell(u, v) \mapsto \ell(u, v)= & \frac{1}{2}\left[-\left|u_{t}\right|^{2}-\left|v_{t}\right|^{2}+|\nabla u|^{2}+|\nabla v|^{2}\right. \\
& \left.+m^{2}|u|^{2}+m^{2}|v|^{2}+F\left(|u|^{2},|v|^{2}\right)\right] .
\end{aligned}
$$

One easily deduces the following multiplier identity from Proposition 2.1:

Proposition 2.4. Assume that $(u(x, t), v(x, t))$ is a smooth solution of (1.2), and $M=h(x, t) \cdot \nabla+h_{n+1} \partial_{t}+q(x, t)$, where $h(x, t)=\left(h_{1}(x, t)\right.$, $\left.\ldots, h_{n}(x, t)\right)$ is a vector function, and $h_{n+1}$ and $q(x, t)$ are scalar functions. Set

$$
\left\{\begin{array}{l}
\operatorname{Eq}_{1}(u, v)=\partial_{t}^{2} u-\Delta u+m^{2} u+F_{1}\left(|u|^{2},|v|^{2}\right) u \\
\operatorname{Eq}_{2}(u, v)=\partial_{t}^{2} v-\Delta v+m^{2} v+F_{2}\left(|u|^{2},|v|^{2}\right) v
\end{array}\right.
$$

Then we have the following general identity:

$$
\begin{aligned}
& (2.19) \quad\left\langle\operatorname{Eq}_{1}(u, v), M u\right\rangle+\left\langle\operatorname{Eq}_{2}(u, v), M v\right\rangle \\
& =\partial_{t}\left\langle\partial_{t} u, h \cdot \nabla u+h_{n+1} \partial_{t} u+q u\right\rangle+\partial_{t}\left\langle\partial_{t} v, h \cdot \nabla v+h_{n+1} \partial_{t} v+q v\right\rangle
\end{aligned}
$$$$
-\nabla \cdot\left\langle\nabla u, h \cdot \nabla u+h_{n+1} \partial_{t} u+q u\right\rangle-\nabla \cdot\left\langle\nabla v, h \cdot \nabla v+h_{n+1} \partial_{t} v+q v\right\rangle
$$$$
+\partial_{t}\left(h_{n+1} \ell(u, v)-\frac{1}{2}|u|^{2} \partial_{t} q-\frac{1}{2}|v|^{2} \partial_{t} q\right)
$$$$
+\nabla \cdot\left(h \ell(u, v)+\frac{1}{2} \nabla q\left(|u|^{2}+|v|^{2}\right)\right)
$$$$
+\frac{1}{2} \square q\left(|u|^{2}+|v|^{2}\right)+\langle\nabla u, \nabla h \nabla u\rangle+\sum_{j=1}^{n} \partial_{j} u \partial_{j} h_{n+1} \partial_{t} u-\sum_{j=1}^{n} \partial_{t} u \partial_{t} h_{j} \partial_{j} u
$$$$
-\partial_{t} u \partial_{t} h_{n+1} \partial_{t} u+\langle\nabla v, \nabla h \nabla v\rangle+\sum_{j=1}^{n} \partial_{j} v \partial_{j} h_{n+1} \partial_{t} v-\sum_{j=1}^{n} \partial_{t} v \partial_{t} h_{j} \partial_{j} v
$$

$$
-\partial_{t} v \partial_{t} h_{n+1} \partial_{t} v+\left(2 q-\nabla \cdot h-\partial_{t} h_{n+1}\right) \ell(u, v)+q \widetilde{F}\left(|u|^{2},|v|^{2}\right),
$$

where

$$
\widetilde{F}\left(|u|^{2},|v|^{2}\right)=F_{1}\left(|u|^{2},|v|^{2}\right)|u|^{2}+F_{2}\left(|u|^{2},|v|^{2}\right)|v|^{2}-F\left(|u|^{2},|v|^{2}\right) .
$$

Choose

$$
M=\frac{x}{r} \cdot \nabla+\frac{n-1}{2 r}, \quad\left(h, h_{n+1}\right)=\left(\frac{x}{r}, 0\right), \quad q(x, t)=\frac{n-1}{2 r} .
$$

Then (2.19) together with (2.14)-(2.17) implies that 


$$
\begin{aligned}
0= & \partial_{t}\left(u_{t} M u\right)+\partial_{t}\left(v_{t} M v\right) \\
& +\nabla \cdot\left\{-\nabla u M u-\nabla v M v+\frac{x}{r} \ell(u, v)-\frac{n-1}{4} \frac{x}{r^{3}}\left[|u|^{2}+|v|^{2}\right]\right\} \\
& +\frac{|\nabla u|^{2}+|\nabla v|^{2}-\left|u_{r}\right|^{2}-\left|v_{r}\right|^{2}}{r}+\frac{(n-1)(n-3)}{4 r^{3}}\left[|u|^{2}+|v|^{2}\right] \\
& +\frac{n-1}{2 r} \widetilde{F}\left(|u|^{2},|v|^{2}\right) .
\end{aligned}
$$

Integrating both sides of (2.21) with respect to $x$ over $\mathbb{R}^{n}$ and applying the divergence theorem, we easily get the following Morawetz-Pohozaev identity for (1.2) with $n \geq 3$.

Corollary 2.5. Let $n \geq 3$. Assume that $(u(x, t), v(x, t))$ is a smooth solution of (1.2), and decays at $|x|=\infty$. Then for $n>3$,

$$
\begin{aligned}
& \frac{(n-1)(n-3)}{4} \int_{\mathbb{R}^{n}} \frac{|u|^{2}+|v|^{2}}{r^{3}} d x \\
& \quad+\int_{\mathbb{R}^{n}} \frac{|\nabla u|^{2}+|\nabla v|^{2}-\left|u_{r}\right|^{2}-\left|v_{r}\right|^{2}}{r} d x \\
& \quad+\frac{n-1}{2} \int_{\mathbb{R}^{n}} \frac{F_{1}\left(|u|^{2},|v|^{2}\right)|u|^{2}+F_{2}\left(|u|^{2},|v|^{2}\right)|v|^{2}-F\left(|u|^{2},|v|^{2}\right)}{r} d x \\
& =-\frac{d}{d t} \int_{\mathbb{R}^{n}}\left[u_{t}\left(u_{r}+\frac{n-1}{2 r} u\right)+v_{t}\left(v_{r}+\frac{n-1}{2 r} v\right)\right] d x,
\end{aligned}
$$

while for $n=3$,

$$
\begin{aligned}
2 \pi|u(0, t)|^{2} & +2 \pi|v(0, t)|^{2}+\int_{\mathbb{R}^{3}} \frac{|\nabla u|^{2}+|\nabla v|^{2}-\left|u_{r}\right|^{2}-\left|v_{r}\right|^{2}}{r} d x \\
& +\int_{\mathbb{R}^{3}} \frac{F_{1}\left(|u|^{2},|v|^{2}\right)|u|^{2}+F_{2}\left(|u|^{2},|v|^{2}\right)|v|^{2}-F\left(|u|^{2},|v|^{2}\right)}{r} d x \\
= & -\frac{d}{d t} \int_{\mathbb{R}^{3}}\left[u_{t}\left(u_{r}+\frac{u}{r}\right)+v_{t}\left(v_{r}+\frac{v}{r}\right)\right] d x .
\end{aligned}
$$

As an immediate consequence of Corollary 2.5, translation invariant with respect to $x$, Hardy's inequality and the conservation of energy, we have the following Morawetz-type estimates.

Corollary 2.6. Let $n \geq 3$, and assume that $(u(x, t), v(x, t))$ is a smooth solution of (1.2), and decays at $|x|=\infty$. Assume that

$$
F_{1}\left(|u|^{2},|v|^{2}\right)|u|^{2}+F_{2}\left(|u|^{2},|v|^{2}\right)|v|^{2}-F\left(|u|^{2},|v|^{2}\right) \geq 0 .
$$


Then

$$
\begin{gathered}
\int_{0}^{\infty} \int_{\mathbb{R}^{n}} \frac{F_{1}\left(|u|^{2},|v|^{2}\right)|u|^{2}+F_{2}\left(|u|^{2},|v|^{2}\right)|v|^{2}-F\left(|u|^{2},|v|^{2}\right)}{r} d x d t \leq C E \\
\int_{0}^{\infty} \int_{\mathbb{R}^{n}} \frac{|\nabla u|^{2}+|\nabla v|^{2}-\left|u_{r}\right|^{2}-\left|v_{r}\right|^{2}}{r} d x d t \leq C E,
\end{gathered}
$$

and

$$
\begin{cases}\int_{0}^{\infty} \int_{\mathbb{R}^{n}} \frac{|u|^{2}+|v|^{2}}{r^{3}} d x d t \leq C E, \quad n>3 \\ \sup _{x} \int_{0}^{\infty}\left[|u|^{2}+|v|^{2}\right] d t \leq C E, \quad n=3\end{cases}
$$

where

$$
E=E\left(u, v, \mathbb{R}^{n}, t\right)=\int_{\mathbb{R}^{n}} e(u, v) d x=E\left(u, v, \mathbb{R}^{n}, 0\right)<\infty
$$

with

$$
\begin{aligned}
e(u, v)= & \frac{1}{2}\left(|\nabla u(x, t)|^{2}+\left|u_{t}(x, t)\right|^{2}+m^{2}|u(x, t)|^{2}\right. \\
& \left.+|\nabla v(x, t)|^{2}+\left|v_{t}(x, t)\right|^{2}+m^{2}|v(x, t)|^{2}+F\left(|u|^{2},|v|^{2}\right)\right) .
\end{aligned}
$$

REMARK 2.1. If $n \leq 2$, we cannot derive the above Morawetz-type estimates because $M u$ is too singular at $x=0$ for $n \leq 2$. Nakanishi constructed in [7] the multiplier $M u=h(x, t) \cdot \mathcal{D}+q(x, t) u$, where

$$
h(x, t)=\frac{(x, t)}{\sqrt{|x|^{2}+t^{2}}} \triangleq \frac{(x, t)}{\lambda}, \quad q=\frac{n-1}{2 \lambda}+\frac{t^{2}-|x|^{2}}{2 \lambda^{3}}, \quad \mathcal{D}=\left(\nabla,-\partial_{t}\right),
$$

and derived some new Morawetz estimates which were used to study the scattering theory for nonlinear Klein-Gordon equations for $n \leq 2$.

If the multiplier operator $M=h \nabla+q$ is a generator of the Poincaré group, we obtain the following identities and conservation laws by Corollary 2.3 or Proposition 2.4.

Corollary 2.7. Let $n \geq 3$. Assume that $(u(x, t), v(x, t))$ is a smooth solution of (1.2), and decays at $|x|=\infty$. Then we have:

(i) (Translation) Setting $M=\partial_{t}$ yields

$$
\begin{aligned}
e(u, v)_{t} & =\nabla \cdot\left\{\partial_{t} u \nabla u+\partial_{t} v \nabla v\right\}, \\
E\left(u, v, \mathbb{R}^{n}, t\right) & =\int_{\mathbb{R}^{n}} e(u, v) d x=E\left(u, v, \mathbb{R}^{n}, 0\right) .
\end{aligned}
$$


Setting $M=\partial_{j}, j=1, \ldots, n$, yields

$$
\begin{gathered}
\left\{-\left|\partial_{j} u\right|^{2}-\left|\partial_{j} v\right|^{2}+\ell(u, v)\right\}_{j}+\sum_{k \neq j}\left\{-\partial_{j} u \partial_{k} u-\partial_{j} v \partial_{k} v\right\}_{k} \\
\quad+\left\{\partial_{j} u \partial_{t} u+\partial_{j} v \partial_{t} v\right\}_{t}=0, \\
\int_{\mathbb{R}^{n}}\left(u_{t} u_{j}+v_{t} v_{j}\right) d x=\text { const. }
\end{gathered}
$$

(ii) (Lorentz transformation) Setting $M=t \partial_{j}+x_{j} \partial_{t}, 1 \leq j \leq n$, gives

$$
\begin{aligned}
& 0= \nabla \cdot\left\{\left(t \partial_{j}+x_{j} \partial_{t}\right) u \nabla u\right\}+\nabla \cdot\left\{\left(t \partial_{j}+x_{j} \partial_{t}\right) v \nabla v\right\} \\
&-\partial_{t}\left\{\left(t \partial_{j}+x_{j} \partial_{t}\right) u \partial_{t} u\right\}-\partial_{t}\left\{\left(t \partial_{j}+x_{j} \partial_{t}\right) v \partial_{t} v\right\} \\
&-\{t \ell(u, v)\}_{j}-\left\{x_{j} \ell(u, v)\right\}_{t}, \\
& \int_{\mathbb{R}^{n}}\left(x_{j} e(u, v)+t u_{t} \partial_{j} u+t v_{t} \partial_{j} v\right) d x=\text { const. }
\end{aligned}
$$

Setting $M=x_{k} \partial_{j}-x_{j} \partial_{k}, 1 \leq j, k \leq n$ with $j \neq k$, yields

$$
\begin{aligned}
& 0= \nabla \cdot\left\{-\left(x_{k} \partial_{j}-x_{j} \partial_{k}\right) u \nabla u\right\}+\nabla \cdot\left\{-\left(x_{k} \partial_{j}-x_{j} \partial_{k}\right) v \nabla v\right\} \\
&+\partial_{t}\left\{\left(x_{k} \partial_{j}-x_{j} \partial_{k}\right) u \partial_{t} u+\left(x_{k} \partial_{j}-x_{j} \partial_{k}\right) v \partial_{t} v\right\} \\
&+\left\{x_{k} \ell(u, v)\right\}_{j}-\left\{x_{j} \ell(u, v)\right\}_{k}, \\
& \int_{\mathbb{R}^{n}}\left(x_{k} \partial_{j}-x_{j} \partial_{k}\right) u \partial_{t} u d x+\int_{\mathbb{R}^{n}}\left(x_{k} \partial_{j}-x_{j} \partial_{k}\right) v \partial_{t} v d x=\text { const. }
\end{aligned}
$$

(iii) (Dilation) Setting $M=x \cdot \nabla+t \partial_{t}+(n-1) / 2$ yields

$$
\begin{aligned}
0= & \frac{n-1}{2}\left[F_{1}\left(|u|^{2},|v|^{2}\right)|u|^{2}+F_{2}\left(|u|^{2},|v|^{2}\right)|v|^{2}\right] \\
& -\frac{n+1}{2} F\left(|u|^{2},|v|^{2}\right)-m^{2}|u|^{2}-m^{2}|v|^{2} \\
& -\nabla \cdot\{M u \nabla u+M v \nabla v+x \ell(u, v)\}+\left\{M u \partial_{t} u+M v \partial_{t} v+t \ell(u, v)_{t} .\right. \\
& \frac{1}{2} \int_{\mathbb{R}^{n}} H(u, v) d x+\frac{d}{d t} \int_{\mathbb{R}^{n}}\left\{M u \partial_{t} u+M v \partial_{t} v+t \ell(u, v)\right\} d x=0,
\end{aligned}
$$

where

$$
\begin{aligned}
H(u, v)= & (n-1)\left[F_{1}\left(|u|^{2},|v|^{2}\right)|u|^{2}+F_{2}\left(|u|^{2},|v|^{2}\right)|v|^{2}\right] \\
& -(n+1) F\left(|u|^{2},|v|^{2}\right)-2 m^{2}\left[|u|^{2}+|v|^{2}\right] .
\end{aligned}
$$

(iv) (Inversion) Setting $M=\left(t^{2}+|x|^{2}\right) \partial_{t}+2 t x \cdot \nabla+(n-1) t$ gives

$$
\begin{aligned}
0= & t H(u, v)-\nabla \cdot\{M u \nabla u+M v \nabla v\}+\partial_{t}\left\{M u \partial_{t} u+M v \partial_{t} v\right\} \\
& +\left\{\left(t^{2}+|x|^{2}\right) \ell(u, v)\right\}_{t}-\frac{n-1}{2}\left[|u|^{2}+|v|^{2}\right]_{t}+\nabla \cdot\{2 t x \ell(u, v)\}
\end{aligned}
$$


and

$$
\begin{aligned}
-t \int_{\mathbb{R}^{n}} H(u, v) d x= & \frac{d}{d t} \int_{\mathbb{R}^{n}}\left\{\left(t^{2}+|x|^{2}\right) e(u, v)+2 \operatorname{tr}\left(u_{r} u_{t}+v_{r} v_{t}\right)\right. \\
& \left.+(n-1) t\left(u u_{t}+v v_{t}\right)-\frac{n-1}{2}\left[|u|^{2}+|v|^{2}\right]\right\} d x .
\end{aligned}
$$

And if for $1 \leq k \leq n$ we set

$$
M=2 x_{k} t \partial_{t}+\left(t^{2}+2 x_{k}^{2}-r^{2}\right) \partial_{k}+2 x_{k} \sum_{j \neq k} x_{j} \partial_{j}+(n-1) x_{k},
$$

then we obtain

$$
\begin{aligned}
0= & x_{k} H(u, v)-\nabla \cdot\{M u \nabla u+M v \nabla v\}+\partial_{t}\left\{M u \partial_{t} u+M v \partial_{t} v\right\} \\
& +\left\{\left(t^{2}+x_{k}^{2}-\sum_{j \neq k} x_{j}^{2}\right) \ell(u, v)\right\}_{k}+\sum_{j \neq k}\left\{2 x_{k} x_{j} \ell(u, v)\right\}_{j} \\
& +\left\{2 x_{k} t \ell(u, v)\right\}_{t}+\frac{n-1}{2}\left\{|u|^{2}+|v|^{2}\right\}_{k}
\end{aligned}
$$

and

$$
\begin{aligned}
0= & \int_{\mathbb{R}^{n}} x_{k} H(u, v) d x+\frac{d}{d t} \int_{\mathbb{R}^{n}}\left\{2 x_{k} t e(u, v)+\left(t^{2}+2 x_{k}^{2}-r^{2}\right)\left(u_{t} \partial_{k} u+v_{t} \partial_{k} v\right)\right. \\
& \left.+2 x_{k} \sum_{j \neq k} x_{j}\left(\partial_{j} u u_{t}+\partial_{j} v v_{t}\right)+(n-1) x_{k} u u_{t}+(n-1) x_{k} v v_{t}\right\} d x
\end{aligned}
$$

3. The decay of local energy in time. Consider the following Cauchy problem for a nonlinear Klein-Gordon system with Hamiltonian structure:

$$
\left\{\begin{array}{l}
u_{t t}-\Delta u+m^{2} u=-F_{1}\left(|u|^{2},|v|^{2}\right) u, \\
v_{t t}-\Delta v+m^{2} v=-F_{2}\left(|u|^{2},|v|^{2}\right) v, \\
u(0)=\varphi_{1}(x), \quad u_{t}(0)=\varphi_{2}(x), \\
v(0)=\psi_{1}(x), \quad v_{t}(0)=\psi_{2}(x),
\end{array}\right.
$$

where there exists a function $F(\lambda, \mu)$ such that

$$
\frac{\partial F(\lambda, \mu)}{\partial \lambda}=F_{1}(\lambda, \mu), \quad \frac{\partial F(\lambda, \mu)}{\partial \mu}=F_{2}(\lambda, \mu)
$$

and

$$
\begin{aligned}
\widetilde{F}\left(|u|^{2},|v|^{2}\right) & =F_{1}\left(|u|^{2},|v|^{2}\right)|u|^{2}+F_{2}\left(|u|^{2},|v|^{2}\right)|v|^{2}-F\left(|u|^{2},|v|^{2}\right) \\
& \geq a F\left(|u|^{2},|v|^{2}\right) \geq 0, \quad a>0 .
\end{aligned}
$$

We have the following decay of local energy in time for (3.1).

THEOREM 3.1. Let $(u(x, t), v(x, t))$ be a smooth solution of (3.1) and (3.2) with 


$$
E\left(u, v, \mathbb{R}^{n}, t\right)=\int_{\mathbb{R}^{n}} e(u, v) d x=E\left(u, v, \mathbb{R}^{n}, 0\right) \triangleq E(\infty)<\infty
$$

where

$$
e(u, v)=\frac{1}{2}\left[\left|u_{t}\right|^{2}+|\nabla u|^{2}+m^{2}|u|^{2}+\left|v_{t}\right|^{2}+|\nabla v|^{2}+m^{2}|v|^{2}+F\left(|u|^{2},|v|^{2}\right)\right] .
$$

Then for any bounded domain $\Omega \subset \mathbb{R}^{n}$,

$$
\begin{gathered}
\lim _{t \rightarrow \infty} \int_{\Omega}\left[|u(x, t)|^{2}+|v(x, t)|^{2}\right] d x=0, \\
\lim _{t \rightarrow \infty} E(u, v, \Omega, t)=\lim _{t \rightarrow \infty} \int_{\Omega} e(u, v) d x=0 .
\end{gathered}
$$

REMARK 3.1. The decay of local energy in time is also valid for mild solutions of (3.1) (i.e. solutions to the associated integral system) because they can be obtained as limits in $C\left(\mathbb{R} ; H^{1}\right) \cap X$, where $X$ denotes a suitable space-time Banach space.

To prove Theorem 3.1, we first establish some necessary estimates on the basis of Morawetz estimates (2.22), (2.23) or their local forms.

Lemma 3.2. Under the assumptions of Theorem 3.1, for $0<T \leq \infty$ we have

$$
\int_{0}^{T}\left[|u(x, t)|^{2}+|v(x, t)|^{2}\right] d t<4 E(\infty), \quad n=3,
$$

and so

$$
\int_{0}^{T} \int_{\Omega}\left[|u(x, t)|^{2}+|v(x, t)|^{2}\right] d x d t \leq C(\Omega) E(\infty), \quad n=3,
$$

and moreover

$$
\begin{gathered}
\int_{0}^{T} \int_{\Omega}\left[|u(x, t)|^{2}+|v(x, t)|^{2}\right] d x d t<C(\Omega) E(\infty), \quad n \geq 4, \\
\int_{0}^{T} E(u, v, \Omega, t) d t<C(\Omega) E(\infty), \quad n \geq 3 .
\end{gathered}
$$

Proof. Choose

$$
\left\{\begin{array}{l}
M u=u_{r}+\frac{n-1}{2 r} u=\frac{x \cdot \nabla u}{r}+\frac{n-1}{2 r} u, \\
M v=v_{r}+\frac{n-1}{2 r} v=\frac{x \cdot \nabla v}{r}+\frac{n-1}{2 r} v,
\end{array}\right.
$$

and multiply the identity $(2.21)$ by $\xi(r)$. It follows that 


$$
\begin{aligned}
0= & \partial_{t}\left(u_{t} M u\right) \xi(r)+\partial_{t}\left(v_{t} M v\right) \xi(r)-\nabla \cdot\left\{\xi ( r ) \left(\nabla u\left(u_{r}+\frac{n-1}{2 r} u\right)\right.\right. \\
& \left.\left.+\nabla v\left(v_{r}+\frac{n-1}{2 r} v\right)-\frac{x}{r} \ell(u, v)+\frac{n-1}{4} \frac{x}{r^{3}}\left[|u|^{2}+|v|^{2}\right]\right)\right\} \\
& +\xi_{r}(r)\left[u_{r}^{2}+v_{r}^{2}+\frac{n-1}{2 r}\left(u_{r} u+v_{r} v\right)-\ell(u, v)\right. \\
& \left.+(n-1) \frac{|u|^{2}+|v|^{2}}{4 r^{2}}\right]+\xi(r) \frac{|\nabla u|^{2}+|\nabla v|^{2}-\left|u_{r}\right|^{2}-\left|v_{r}\right|^{2}}{r} \\
& +\frac{(n-1)(n-3)}{4 r^{3}} \xi(r)\left[|u|^{2}+|v|^{2}\right]+\frac{n-1}{2 r} \xi(r) \widetilde{F}\left(|u|^{2},|v|^{2}\right),
\end{aligned}
$$

where $\ell(u, v)$ and $\widetilde{F}\left(|u|^{2},|v|^{2}\right)$ are defined by (2.18) and (2.20) respectively.

Without loss of generality, we only prove Lemma 3.2 for the initial data with compact support, i.e.,

$$
\operatorname{supp} \varphi_{j} \subset\{x:|x| \leq k\}, \quad \operatorname{supp} \psi_{j} \subset\{x:|x| \leq k\}, \quad j=1,2,
$$

for some $k \in \mathbb{N}$. In fact, let $t_{0}>0$ and for any $x_{0} \in \mathbb{R}^{n}$, put

$$
\Lambda\left(x_{0}, t_{0}\right)=\left\{(x, t) \in \mathbb{R}^{n+1}:\left|x-x_{0}\right| \leq t_{0}-t, 0 \leq t \leq t_{0}\right\},
$$

the backward light cone through $\left(x_{0}, t_{0}\right)$. Then $(u(x, t), v(x, t))$ in $\Lambda\left(x_{0}, t_{0}\right)$ depends only on the initial data $\varphi_{1}, \psi_{1}, \varphi_{2}$ and $\psi_{2}$ on the ball $B_{t_{0}}\left(x_{0}\right)=$ $\left\{(x, 0):\left|x-x_{0}\right| \leq t_{0}\right\}$.

Let $\left(u^{*}(x, t), v^{*}(x, t)\right)$ be a solution of (3.1) with

$$
\left\{\begin{array}{l}
\operatorname{supp} u^{*}(\cdot, 0), \operatorname{supp} u_{t}^{*}(\cdot, 0) \subset\left\{x:\left|x-x_{0}\right| \leq 2 t_{0}\right\} \\
\operatorname{supp} v^{*}(\cdot, 0), \operatorname{supp} v_{t}^{*}(\cdot, 0) \subset\left\{x:\left|x-x_{0}\right| \leq 2 t_{0}\right\}
\end{array}\right.
$$

and

$$
\left\{\begin{array}{lll}
u^{*}(x, 0)=u(x, 0), & u_{t}^{*}(x, 0)=u_{t}(x, 0), & x \in B_{t_{0}}\left(x_{0}\right), \\
v^{*}(x, 0)=v(x, 0), & v_{t}^{*}(x, 0)=v_{t}(x, 0), & x \in B_{t_{0}}\left(x_{0}\right)
\end{array}\right.
$$

and

$$
E^{*}(\infty)<(1+\varepsilon) E(\infty)
$$

where

$$
\lim _{t_{0} \rightarrow \infty} \varepsilon=0 .
$$

Then $(u(x, t), v(x, t))=\left(u^{*}(x, t), v^{*}(x, t)\right)$ in $\Lambda\left(x_{0}, t_{0}\right)$. Since Lemma 3.2 is valid for $\left(u^{*}(x, t), v^{*}(x, t)\right)$, it follows that

$$
\begin{aligned}
\int_{0}^{t_{0}}\left[\left|u\left(x_{0}, t\right)\right|^{2}+\left|v\left(x_{0}, t\right)\right|^{2}\right] d t & =\int_{0}^{t_{0}}\left[\left|u^{*}\left(x_{0}, t\right)\right|^{2}+\left|v^{*}\left(x_{0}, t\right)\right|^{2}\right] d t \\
& <4 E^{*}(\infty)<4(1+\varepsilon) E(\infty) .
\end{aligned}
$$

Letting $t_{0} \rightarrow \infty$ on both sides of (3.10), one easily sees that 


$$
\int_{0}^{\infty}\left[\left|u\left(x_{0}, t\right)\right|^{2}+\left|v\left(x_{0}, t\right)\right|^{2}\right] d t \leq 4 E(\infty), \quad \forall x_{0} \in \mathbb{R}^{n} .
$$

A similar argument establishes (3.7).

On the other hand, for any bounded domain $\Omega \subset \mathbb{R}^{n}$, we can choose $t_{0}>0$ sufficiently large such that $\Omega \subset B_{t_{0} / 2}\left(x_{0}\right)$. Due to the finite propagation speed, it follows that

$$
\begin{aligned}
\int_{0}^{t_{0} / 2} E(u, v, \Omega, t) d t & =\int_{0}^{t_{0} / 2} E\left(u^{*}, v^{*}, \Omega, t\right) d t<C(\Omega) E^{*}(\infty) \\
& <C(\Omega) E(\infty)(1+\varepsilon) .
\end{aligned}
$$

Letting $t_{0} \rightarrow \infty$ on both sides of (3.11) yields (3.8).

Now we begin to prove the special case of the lemma. Integrating both sides of (2.22) and (2.23) with respect to time $t$ from 0 to $T$, one gets for $n=3$,

$$
\begin{array}{r}
2 \pi \int_{0}^{T}\left[|u(x, t)|^{2}+|v(x, t)|^{2}\right] d t+\int_{0}^{T} \int_{\mathbb{R}^{3}} \frac{|\nabla u|^{2}-\left|u_{r}\right|^{2}+|\nabla v|^{2}-\left|v_{r}\right|^{2}}{r} d x d t \\
\quad+\int_{0}^{T} \int_{\mathbb{R}^{3}} \frac{\widetilde{F}\left(|u|^{2},|v|^{2}\right)}{r} d x d t \leq 4 E(\infty), \quad 0<T \leq \infty
\end{array}
$$

while for $n \geq 4$,

$$
\begin{aligned}
& \frac{(n-1)(n-3)}{4} \int_{0}^{T} \int_{\mathbb{R}^{n}} \frac{|u|^{2}+|v|^{2}}{r^{3}} d x d t \\
& +\int_{0}^{T} \int_{\mathbb{R}^{n}} \frac{|\nabla u|^{2}+|\nabla v|^{2}-\left|u_{r}\right|^{2}-\left|v_{r}\right|^{2}}{r} d x d t \\
& +\frac{n-1}{2} \int_{0}^{T} \int_{\mathbb{R}^{n}} \frac{\widetilde{F}\left(|u|^{2},|v|^{2}\right)}{r} d x d t \leq 4 E(\infty), \quad 0<T \leq \infty
\end{aligned}
$$

by (3.3) together with the Hardy inequalities

$$
\int_{\mathbb{R}^{n}}\left|\frac{u}{r}\right|^{2} d x \leq\|\nabla u\|_{2}^{2}, \quad \int_{\mathbb{R}^{n}}\left|\frac{v}{r}\right|^{2} d x \leq\|\nabla v\|_{2}^{2} .
$$

Since

$$
|\nabla u|^{2}-\left|u_{r}\right|^{2}+|\nabla v|^{2}-\left|v_{r}\right|^{2} \geq 0, \quad \widetilde{F}\left(|u|^{2},|v|^{2}\right) \geq a F\left(|u|^{2},|v|^{2}\right) \geq 0,
$$

we see that (3.6) holds by (3.12).

For any bounded domain $\Omega \subset \mathbb{R}^{n}$, set $\varrho=\operatorname{dist}(0, \Omega)+|\Omega|>0$. One easily sees by (3.13) that for $n \geq 4$, 


$$
\int_{0}^{T} \int_{\Omega} \frac{|u|^{2}+|v|^{2}}{\varrho^{3}} d x d t \leq \int_{0}^{T} \int_{\Omega} \frac{|u|^{2}+|v|^{2}}{r^{3}} d x d t \leq \frac{16}{(n-1)(n-3)} E(\infty) .
$$

Thus we obtain (3.7).

We still need to prove (3.8). For any bounded domain $\Omega \subset \mathbb{R}^{n}$, a similar argument implies that for $n \geq 3$,

$$
\begin{aligned}
\int_{0}^{T} \int_{\Omega}\left(|\nabla u|^{2}+\right. & \left.|\nabla v|^{2}-\left|u_{r}\right|^{2}-\left|v_{r}\right|^{2}\right) d x d t \\
& +\frac{n-1}{2} \int_{0}^{T} \int_{\Omega} \widetilde{F}\left(|u|^{2},|v|^{2}\right) d x d t \leq 4 \varrho E(\infty), \quad 0<T \leq \infty,
\end{aligned}
$$

by (3.12) and (3.13). Setting $\Omega_{j k}=x_{k} \partial_{j}-x_{j} \partial_{k}$, it follows that

$$
|\nabla u|^{2}-\left|u_{r}\right|^{2}=\left|\nabla u-\frac{x}{|x|}\left(\frac{x}{|x|} \cdot \nabla u\right)\right|^{2}=\frac{1}{r^{2}} \sum_{j}\left|\sum_{k} x_{k} \Omega_{j k} u\right|^{2} .
$$

From the invariance of (3.1) under the translation and rotation transformation groups, we obtain

$$
\begin{aligned}
& \int_{0}^{T} \int_{\Omega}\left|\nabla_{x-y} u-\frac{x-y}{|x-y|}\left(\frac{x-y}{|x-y|} \cdot \nabla_{x-y} u\right)\right|^{2} d x d t \leq C(\Omega) E(\infty), \quad y \in \mathbb{R}^{n}, \\
& \int_{0}^{T} \int_{\Omega}\left|\nabla_{x-y} v-\frac{x-y}{|x-y|}\left(\frac{x-y}{|x-y|} \cdot \nabla_{x-y} v\right)\right|^{2} d x d t \leq C(\Omega) E(\infty), \quad y \in \mathbb{R}^{n} .
\end{aligned}
$$

Choose three different points $y, z, w \in B_{1}(0)$ which are not collinear. For continuous vector fields $\nabla u$ and $\nabla v$ on the bounded domain $\Omega$, we always have

where

$$
\begin{aligned}
\nabla u(x, t)= & \alpha_{1}(x)\left(\nabla_{x-y} u-\frac{x-y}{|x-y|}\left(\frac{x-y}{|x-y|} \cdot \nabla_{x-y} u\right)\right) \\
& +\beta_{1}(x)\left(\nabla_{x-z} u-\frac{x-z}{|x-z|}\left(\frac{x-z}{|x-z|} \cdot \nabla_{x-z} u\right)\right) \\
& +\gamma_{1}(x)\left(\nabla_{x-w} u-\frac{x-w}{|x-w|}\left(\frac{x-w}{|x-w|} \cdot \nabla_{x-w} u\right)\right), \\
\nabla v(x, t)= & \alpha \alpha_{2}(x)\left(\nabla_{x-y} v-\frac{x-y}{|x-y|}\left(\frac{x-y}{|x-y|} \cdot \nabla_{x-y} v\right)\right) \\
& +\beta_{2}(x)\left(\nabla_{x-z} v-\frac{x-z}{|x-z|}\left(\frac{x-z}{|x-z|} \cdot \nabla_{x-z} v\right)\right) \\
& +\gamma_{2}(x)\left(\nabla_{x-w} v-\frac{x-w}{|x-w|}\left(\frac{x-w}{|x-w|} \cdot \nabla_{x-w} u\right)\right),
\end{aligned}
$$

$$
\left|\alpha_{j}(x)\right|,\left|\beta_{j}(x)\right|,\left|\gamma_{j}(x)\right| \leq C, \quad j=1,2, x \in \Omega .
$$


Thus for $0<T \leq \infty$ and $n \geq 3$,

$$
\int_{0}^{T} \int_{\Omega}\left(|\nabla u|^{2}+|\nabla v|^{2}\right) d x d t+a \int_{0}^{T} \int_{\Omega} F\left(|u|^{2},|v|^{2}\right) d x d t \leq 4 C E(\infty) .
$$

We still have to estimate $\left\|u_{t}\right\|_{L^{2}(\Omega)},\|u\|_{L^{2}(\Omega)},\left\|v_{t}\right\|_{L^{2}(\Omega)},\|v\|_{L^{2}(\Omega)}$ to obtain (3.8). Integrating (3.9) with respect to $(x, t) \in \mathbb{R}^{n} \times[0, T]$, we obtain by the divergence theorem, for $n \geq 4$,

$$
\begin{aligned}
& \quad \int_{0}^{T} \int_{\mathbb{R}^{n}} \xi_{r}(r)\left[\frac{\left|u_{t}\right|^{2}}{2}+\frac{(n-1)|u|^{2}}{4 r^{2}}-\frac{m^{2}|u|^{2}}{2}+\frac{n-1}{2} \frac{u u_{r}}{r}\right. \\
& \left.+\frac{\left|v_{t}\right|^{2}}{2}+\frac{(n-1)|v|^{2}}{4 r^{2}}-\frac{m^{2}|v|^{2}}{2}+\frac{n-1}{2} \frac{v v_{r}}{r}\right] d x d t \\
= & \int_{0}^{T} \int_{\mathbb{R}^{n}} \xi_{r}(r)\left[\frac{|\nabla u|^{2}}{2}+\frac{|\nabla v|^{2}}{2}+\frac{1}{2} F\left(|u|^{2},|v|^{2}\right)-\left|u_{r}\right|^{2}-\left|v_{r}\right|^{2}\right] d x d t \\
& -\int_{0}^{T} \int_{\mathbb{R}^{n}} \xi(r)\left[\frac{|\nabla u|^{2}+|\nabla v|^{2}-\left|u_{r}\right|^{2}-\left|v_{r}\right|^{2}}{r}+\frac{(n-1)(n-3)}{4 r^{3}}\left[|u|^{2}+|v|^{2}\right]\right. \\
& \left.+\frac{n-1}{2 r} \widetilde{F}\left(|u|^{2},|v|^{2}\right)\right] d x d t-\left.\int_{\mathbb{R}^{n}} \xi(r) \frac{2 r u_{r}+(n-1) u}{2 r} u_{t} d x\right|_{0} ^{T} \\
& -\left.\int_{\mathbb{R}^{n}} \xi(r) \frac{2 r v_{r}+(n-1) v}{2 r} v_{t} d x\right|_{0} ^{T},
\end{aligned}
$$

while for $n=3$,

$$
\begin{aligned}
& \int_{0}^{T} \int_{\mathbb{R}^{3}} \xi_{r}(r)\left[\frac{\left|u_{t}\right|^{2}+\left|v_{t}\right|^{2}}{2}+\frac{|u|^{2}+|v|^{2}}{2 r^{2}}-m^{2} \frac{|u|^{2}+|v|^{2}}{2}+\frac{u u_{r}+v v_{r}}{r}\right] d x d t \\
& =\int_{0}^{T} \int_{\mathbb{R}^{3}} \xi_{r}(r)\left[\frac{|\nabla u|^{2}}{2}+\frac{|\nabla v|^{2}}{2}+\frac{1}{2} F\left(|u|^{2},|v|^{2}\right)-\left|u_{r}\right|^{2}-\left|v_{r}\right|^{2}\right] d x d t \\
& \quad-\int_{0}^{T} \int_{\mathbb{R}^{3}} \xi(r)\left[\frac{|\nabla u|^{2}+|\nabla v|^{2}-\left|u_{r}\right|^{2}-\left|v_{r}\right|^{2}+\widetilde{F}\left(|u|^{2},|v|^{2}\right)}{r}\right] d x d t \\
& \quad-\left.\int_{\mathbb{R}^{3}} \xi(r) \frac{r u_{r}+u}{r} u_{t} d x\right|_{0} ^{T}-\left.\int_{\mathbb{R}^{3}} \xi(r) \frac{r v_{r}+v}{r} v_{t} d x\right|_{0} ^{T} \\
& \quad-2 \pi \int_{0}^{T} \xi(r)\left[|u(x, t)|^{2}+|v(x, t)|^{2}\right] d t .
\end{aligned}
$$


We choose $\xi(r)$ to be a continuous function such that

$$
\xi_{r}(r)= \begin{cases}-1, & r \leq r_{0}, \\ 0, & r>r_{0},\end{cases}
$$

and set $D=\left\{x: r=\left|x-x_{0}\right| \leq r_{0}\right\}, r_{0}>0$.

Adding

$$
(n-1) \int_{0}^{T} \int_{\mathbb{R}^{n}} \xi_{r}(r)\left|u_{r}\right|^{2} d x d t+(n-1) \int_{0}^{T} \int_{\mathbb{R}^{n}} \xi_{r}(r)\left|v_{r}\right|^{2} d x d t
$$

to both sides of (3.15) or (3.16) and writing, for $n \geq 3$,

$$
\begin{aligned}
&(n-1) {\left[\left|u_{r}\right|^{2}+\left|v_{r}\right|^{2}\right]+\frac{n-1}{2} \frac{u u_{r}+v v_{r}}{r}+\frac{n-1}{4} \frac{|u|^{2}+|v|^{2}}{r^{2}} } \\
&=(n-1)\left[\left(u_{r}+\frac{u}{4 r}\right)^{2}+\left(v_{r}+\frac{v}{4 r}\right)^{2}\right]+\frac{3(n-1)}{16} \frac{|u|^{2}+|v|^{2}}{r^{2}},
\end{aligned}
$$

it follows that for $n \geq 4$,

$$
\begin{aligned}
& \int_{0 \mathbb{R}^{n}}^{T} \int_{r} \xi_{r}(r)\left[\frac{\left|u_{t}\right|^{2}}{2}+\frac{3(n-1)|u|^{2}}{16 r^{2}}-\frac{m^{2}|u|^{2}}{2}+(n-1)\left(u_{r}+\frac{u}{4 r}\right)^{2}\right. \\
& \left.\quad+\frac{\left|v_{t}\right|^{2}}{2}+\frac{3(n-1)|v|^{2}}{16 r^{2}}-\frac{m^{2}|v|^{2}}{2}+(n-1)\left(v_{r}+\frac{v}{4 r}\right)^{2}\right] d x d t \\
& =\int_{0}^{T} \int_{\mathbb{R}^{n}} \xi_{r}(r)\left[\frac{|\nabla u|^{2}}{2}+\frac{|\nabla v|^{2}}{2}+\frac{1}{2} F\left(|u|^{2},|v|^{2}\right)+(n-2)\left(\left|u_{r}\right|^{2}+\left|v_{r}\right|^{2}\right)\right] d x d t \\
& \quad-\int_{0}^{T} \xi(r)\left[\frac{|\nabla u|^{2}+|\nabla v|^{2}-\left|u_{r}\right|^{2}-\left|v_{r}\right|^{2}}{r}+\frac{(n-1)(n-3)}{4 r^{3}}\left[|u|^{2}+|v|^{2}\right]\right. \\
& \left.\quad+\frac{n-1}{2 r} \widetilde{F}\left(|u|^{2},|v|^{2}\right)\right] d x d t-\left.\int_{\mathbb{R}^{n}} \xi(r) \frac{2 r u_{r}+(n-1) u}{2 r} u_{t} d x\right|_{0} ^{T} \\
& \quad-\left.\int_{\mathbb{R}^{n}} \xi(r) \frac{2 r v_{r}+(n-1) v}{2 r} v_{t} d x\right|_{0} ^{T},
\end{aligned}
$$

while for $n=3$,

$$
\begin{aligned}
\int_{0}^{T} \int_{\mathbb{R}^{3}} \xi_{r}(r)\left[\frac{\left|u_{t}\right|^{2}}{2}+\frac{3|u|^{2}}{8 r^{2}}\right. & -\frac{m^{2}|u|^{2}}{2}+2\left(u_{r}+\frac{u}{4 r}\right)^{2} \\
& \left.+\frac{\left|v_{t}\right|^{2}}{2}+\frac{3|v|^{2}}{8 r^{2}}-\frac{m^{2}|v|^{2}}{2}+2\left(v_{r}+\frac{v}{4 r}\right)^{2}\right] d x d t
\end{aligned}
$$




$$
\begin{aligned}
= & \int_{0}^{T} \int_{\mathbb{R}^{3}} \xi_{r}(r)\left[\frac{|\nabla u|^{2}}{2}+\frac{|\nabla v|^{2}}{2}+\frac{1}{2} F\left(|u|^{2},|v|^{2}\right)+\left(\left|u_{r}\right|^{2}+\left|v_{r}\right|^{2}\right)\right] d x d t \\
& -\int_{0}^{T} \int_{\mathbb{R}^{3}} \xi(r)\left[\frac{|\nabla u|^{2}+|\nabla v|^{2}-\left|u_{r}\right|^{2}-\left|v_{r}\right|^{2}+\widetilde{F}\left(|u|^{2},|v|^{2}\right)}{r}\right] d x d t \\
& -\left.\int_{\mathbb{R}^{3}} \xi(r) \frac{r u_{r}+u}{r} u_{t} d x\right|_{0} ^{T}-\left.\int_{\mathbb{R}^{3}} \xi(r) \frac{r v_{r}+v}{r} v_{t} d x\right|_{0} ^{T} \\
& -2 \pi \int_{0}^{T} \xi(r)|u(x, t)|^{2} d t-2 \pi \int_{0}^{T} \xi(r)|v(x, t)|^{2} d t .
\end{aligned}
$$

Choose $r_{0}=\sqrt{3(n-1)} / 4 m$. Then for $n \geq 3$,

$$
\int_{0}^{T} \int_{D}\left(u_{t}^{2}+v_{t}^{2}+\frac{3(n-1)}{32 r^{2}}|u|^{2}+\frac{3(n-1)}{32 r^{2}}|v|^{2}\right) d x d t \leq C_{0} E(\infty) .
$$

Combining (3.14) with (3.17), it follows that

$$
\int_{0}^{T} E(u, v, D, t) d t \leq C_{1} E(\infty) .
$$

Note that any bounded region $\Omega$ can be covered by a finite number $k$ of disks $D$. By adding the corresponding inequalities we obtain (3.8) with $C(\Omega)=k C_{1}$ depending only on $\Omega$.

Proof of Theorem 3.1. It is easy to verify

$$
\begin{aligned}
\left(t-t_{1}\right) \int_{\Omega} u^{2} d x & =\int_{t_{1}}^{t}\left[\left(\tau-t_{1}\right) \int_{\Omega} u^{2} d x\right]_{\tau} d \tau \\
& =\int_{t_{1}}^{t} \int_{\Omega} u^{2} d x d \tau+2 \int_{t_{1}}^{t} \int_{\Omega}\left(\tau-t_{1}\right) u u_{\tau} d x d \tau \\
& \leq 2 \int_{t_{1}}^{t} \int_{\Omega}|u|^{2} d x d \tau+\int_{t_{1}} \int_{\Omega}\left(\tau-t_{1}\right)^{2}\left|u_{\tau}\right|^{2} d x d \tau
\end{aligned}
$$

by the Newton-Leibniz formula and Hölder's inequality. A similar argument implies that

$$
\left(t-t_{1}\right) \int_{\Omega} v^{2} d x \leq 2 \int_{t_{1}}^{t} \int_{\Omega}|v|^{2} d x d \tau+\int_{t_{1}}^{t} \int_{\Omega}\left(\tau-t_{1}\right)^{2}\left|v_{\tau}\right|^{2} d x d \tau
$$

Set $t_{1}=t-1$ and note that

$$
\int_{0}^{\infty} \int_{\Omega}\left[|u(x, t)|^{2}+|v(x, t)|^{2}\right] d x d t<C(\Omega) E(\infty) .
$$


It follows that

$$
\lim _{t \rightarrow \infty} \int_{\Omega}\left[|u(x, t)|^{2}+|v(x, t)|^{2}\right] d x=0 .
$$

To prove (3.5), we need the following claim:

Claim. If $f(t) \geq 0, \sup _{t>0}\left|f^{\prime}(t)\right|<\infty$, and $\int_{0}^{\infty} f(t) d t<\infty$, then

$$
\lim _{t \rightarrow \infty} f(t)=0 \text {. }
$$

In fact, if not, then for fixed $\varepsilon_{0}>0$ and all $T>0$, there exists a $t>T$ such that $f(t) \geq \varepsilon_{0}$. Since

$$
\sup _{t}\left|f^{\prime}(t)\right|=C<\infty \Rightarrow|f(t)-f(s)| \leq C|t-s|,
$$

one easily sees that

$$
\begin{array}{cll}
\text { for } \quad T_{1}=\frac{2 \varepsilon_{0}}{C}, & \exists t_{1}>T_{1}, & f\left(t_{1}\right) \geq \varepsilon_{0}, \\
\text { for } \quad T_{2}=t_{1}+\frac{2 \varepsilon_{0}}{C}, & \exists t_{2}>T_{2}, & f\left(t_{2}\right) \geq \varepsilon_{0}, \\
\vdots & \\
\text { for } \quad T_{k}=t_{k-1}+\frac{2 \varepsilon_{0}}{C}, & \exists t_{k}>T_{k}, & f\left(t_{k}\right) \geq \varepsilon_{0},
\end{array}
$$

In view of the definition of continuous function, for any $t \in I_{k}=\left[t_{k}-\right.$ $\left.\varepsilon_{0} / 2 C, t_{k}+\varepsilon_{0} / 2 C\right]$, we have

$$
|f(t)| \geq\left|f\left(t_{k}\right)\right|-\left|f(t)-f\left(t_{k}\right)\right| \geq \varepsilon_{0}-C \frac{\varepsilon_{0}}{2 C}=\frac{\varepsilon_{0}}{2} .
$$

Thus

$$
\int_{0}^{\infty} f(t) d t \geq \sum_{k}\left(\min _{t \in I_{k}}|f(t)|\right)\left|I_{k}\right| \geq \sum_{k} \frac{\varepsilon_{0}^{2}}{2 C}=\infty .
$$

This is a contradiction, completing the proof of the Claim.

Denote by $\Omega(\varrho)$ the set of disks with centers $x_{0}$ and radius $\varrho$, and define

$$
G(t)=\int_{\varrho_{1}}^{\varrho_{2}} E(u, v, \Omega(\varrho), t) d \varrho .
$$

Thus

$$
\begin{aligned}
\int_{0}^{T} G(t) d t & =\int_{0}^{T} \int_{\varrho_{1}}^{\varrho_{2}} E(u, v, \Omega(\varrho), t) d \varrho d t \leq E(\infty) \int_{\varrho_{1}}^{\varrho_{2}} C_{\varrho} d \varrho \\
& \leq E(\infty) C_{\max }\left(\varrho_{2}-\varrho_{1}\right) .
\end{aligned}
$$

On the other hand, note that 


$$
\begin{aligned}
E_{t}= & \int_{\Omega}\left(u_{t} u_{t t}+\nabla u \cdot \nabla u_{t}+m^{2} u u_{t}+v_{t} v_{t t}+\nabla v \cdot \nabla v_{t}+m^{2} v v_{t}\right. \\
& \left.+F_{1}\left(|u|^{2},|v|^{2}\right) u u_{t}+F_{2}\left(|u|^{2},|v|^{2}\right) v v_{t}\right) d x \\
= & \int_{\Omega}\left(u_{t} \Delta u+\nabla u \cdot \nabla u_{t}+v_{t} \Delta v+\nabla v \cdot \nabla v_{t}\right) d x \\
= & \int_{\Omega}\left[\nabla \cdot\left(u_{t} \nabla u\right)+\nabla \cdot\left(v_{t} \nabla v\right)\right] d x=\int_{\left|x-x_{0}\right|=\varrho}\left[u_{r} u_{t}+v_{r} v_{t}\right] d \sigma .
\end{aligned}
$$

We obtain

$$
\begin{aligned}
G_{t} & =\int_{\varrho_{1}}^{\varrho_{2}} E_{t}(u, v, \Omega(\varrho), t) d \varrho=\int_{\varrho_{1}}^{\varrho_{2}} \int_{\left|x-x_{0}\right|=\varrho}\left[u_{r} u_{t}+v_{r} v_{t}\right] d \sigma d \varrho \\
& =\int_{\varrho_{1} \leq\left|x-x_{0}\right| \leq \varrho_{2}}\left[u_{t} u_{r}+v_{t} v_{r}\right] d x \leq \frac{1}{2} \int_{\mathbb{R}^{n}}\left(u_{t}^{2}+u_{r}^{2}+v_{t}^{2}+v_{r}^{2}\right) d x \leq E(\infty) .
\end{aligned}
$$

This together with the Claim implies that

$$
\lim _{t \rightarrow \infty} G(t)=0
$$

Since $E \geq 0$, we get

$$
\lim _{t \rightarrow \infty} E(u, v, \Omega(\varrho), t) \leq \lim _{t \rightarrow \infty} \frac{1}{\varrho_{2}-\varrho_{1}} G(t)=0 .
$$

This completes the proof of Theorem 3.1.

REMARK 3.2. $L^{2}$-decay (3.4) is an immediate consequence of the estimates

$$
\begin{gathered}
\int_{0}^{\infty} \int_{\Omega}\left[|u(x, t)|^{2}+|v(x, t)|^{2}\right] d x d t<C(\Omega) E(\infty), \\
\frac{d}{d t} \int_{\Omega}\left[|u(x, t)|^{2}+|v(x, t)|^{2}\right] d x \leq \int_{\Omega}\left(\left|u_{t}\right|^{2}+|\nabla u|^{2}+\left|v_{t}\right|^{2}+|\nabla v|^{2}\right) d x<\infty .
\end{gathered}
$$

Acknowledgements. The research of C. X. Miao was partly supported by the NSF of China, No. 10571016, and the Institute of Mathematical Sciences, Chinese University of Hong Kong. He wishes to thank Prof. Zhouping Xin for the stimulating discussion about this problem.

\section{References}

[1] P. Brenner, On scattering and everywhere defined scattering operator for nonlinear Klein-Gordon equations, J. Differential Equations 56 (1985), 310-344.

[2] J. Ginibre and G. Velo, Conformal invariance and time decay for nonlinear wave equations, Ann. Inst. H. Poincaré Phys. Théorique 47 (1987), 221-276. 
[3] J. Ginibre and G. Velo, Scattering theory in energy space for a class of nonlinear Schrödinger equations, J. Math. Pures Appl. 64 (1985), 363-401.

[4] J. E. Lin and W. Strauss, Decay and scattering of Schödinger equation, J. Funct. Anal. 30 (1978), 245-263.

[5] C. Morawetz, Time decay for nonlinear Klein-Gordon equations, Proc. Roy. Soc. London A 306 (1968), 503-518.

[6] C. S. Morawetz and W. Strauss, Decay and scattering of solutions of a nonlinear relativistic wave equation, Comm. Pure Appl. Math. 25 (1972), 1-31.

[7] K. Nakanishi, Remarks on the energy scattering for nonlinear Klein-Gordon and Schrödinger equations, Tohoku Math. J. 53 (2001), 285-303.

[8] W. Strauss, Nonlinear invariant wave equations, in: Invariant Wave Equations, Lecture Notes in Phys. 78, Springer, 1978, 197-249.

[9] —, Nonlinear Wave Equations, CBMS Reg. Conf. Ser. Math. 73, Amer. Math. Soc., 1989.

Institute of Applied Physics

and Computational Mathematics

P.O. Box 8009

Beijing 100088, China

E-mail: miao_changxing@iapcm.ac.cn
Department of Mathematics

Xidian University

P.O. Box 245

Xi'an, Shanxi 710071, China

E-mail: youbinzhu@yahoo.com.cn 